\title{
Crecimiento económico de América Latina en el siglo XX
}

Miller Rivera Lozano Universidad Santo Tomás

Colombia

Nicolás Rivera Garzón

Universidad Nacional de Colombia

Colombia 


\title{
Crecimiento económico de América Latina en el siglo XX
}

\author{
Miller Rivera Lozano ${ }^{1}$ y Nicolás Rivera Garzón ${ }^{2}$ (D) \\ ${ }^{1}$ Universidad Santo Tomás - Colombia \\ 2 Universidad Nacional de Colombia - Colombia
}

\section{RESUMEN}

El objetivo de este artículo es determinar el impacto de los factores: crecimiento del stock de capital, formación bruta de capital, crecimiento de la población, gasto de consumo final del gobierno general, nivel de inflación y exportaciones de bienes y servicios en el crecimiento económico de América Latina durante el periodo de 1900 a 2000. Los países de la muestra son Argentina, Bolivia, Brasil, Chile, Colombia, Costa Rica, Cuba, República Dominicana, Ecuador, El Salvador, Guatemala, Haití, Honduras, México, Nicaragua, Panamá, Paraguay, Perú, Uruguay, Venezuela. Los resultados indican que el crecimiento del stock de capital y la formación bruta de capital son variables centrales para explicar el proceso de acumulación de capital y el aumento de capacidad productiva en cualquiera de las economías. Además, el gasto del gobierno general como proporción del PIB, mostró una relación negativa con el crecimiento del ingreso real per cápita; sin embargo, el gasto público es clave para solucionar conflictos distributivos y sociales. Finalmente, el comercio exterior es clave para que cualquier país logre beneficiarse de las ventajas comparativas, financiamiento exterior, flujo de ideas y de bienes del resto del mundo.

Palabras clave: crecimiento económico; macroeconomía; Latinoamérica; ingreso por habitante.

JEL: C21, E22, F10, H30, N16.

Editor: Andrés Escobar Espinoza Universidad de Cartagena, Colombia.

Tipología IBN Publindex: Artículo de investigación científica y tecnológica

Copyright: (C) 2020. Rivera, M. y Rivera, N. Este es un artículo de acceso abierto, distribuido bajo los términos de la licencia https://creativecommons.org/licenses/bync-sa/4.0/ la cual permite el uso sin restricciones, distribución y reproducción en cualquier medio, siempre y cuando que el original, el autor y la fuente sean acreditados.

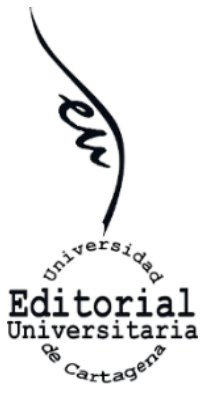

\section{Latin America's economic growth during the 20th century}

\section{ABSTRACT}

The objective of this article is to determine the impact of these factors on the economic growth of Latin America during the period from 1900 to 2000: capital stock growth, gross capital formation, population growth, final consumption expenditure of the central government, inflation and exports of goods and services. The countries in the sample are Argentina, Bolivia, Brazil, Chile, Colombia, Costa Rica, Cuba, Dominican Republic, Ecuador, El Salvador, Guatemala, Haiti, Honduras, Mexico, Nicaragua, Panama, Paraguay, Peru, Uruguay, Venezuela. The results indicate that the growth of the capital stock and gross capital formation are central variables to explain the process of capital accumulation and the increase in productive capacity in any of the economies. In addition, government spending as a proportion of GDP showed a negative relationship with the growth of real income per capita; however, public spending is key to solving distributional and social conflicts. Finally, foreign trade is key for any country to be able to benefit from comparative advantages, foreign financing, flow of ideas and goods from the rest of the world.

Keywords: Economic growth; macroeconomics; Latin America; income per capita.

JEL: C21, E22, F10, H30, N16. 


\section{INTRODUCCIÓN}

La literatura sobre crecimiento económico se caracteriza por tener numerosos e interesantes enfoques para responder la pregunta de ¿Cómo crecen los países?; y, por ende, ¿Cómo mejorar el desempeño económico de las democracias contemporáneas? Dentro de dicha diversidad, se pueden clasificar la mayoría de las teorías dentro de tres grupos: crecimiento por acumulación de factores, crecimiento por innovaciones e hipótesis institucionales. En el primer grupo se encuentran los trabajos de Solow (1956), Cass (1965), Koopmans (1965), Romer (1990) y Aghion \& Howitt (1992). El crecimiento por innovaciones es explicado por Schumpeter (1911), Young (1928), Currie \& Sandilands (1997) y Lorente (2018). Mientras que las principales hipótesis institucionalistas se encuentran en Acemoğlu, Johnson, \& Robinson (2001), Engerman \& Sokoloff (2002), Nunn (2009) y Acemoğlu \& Robinson (2010). Asimismo, abundan los trabajos cuyo objetivo es contrastar, probar o refutar estas teorías mediante el uso de paneles de datos de países; los principales aportes son Wolf (1994), Mankiw, Phelps, \& Romer (1995), Slemrod, Gale, \& Easterly (1995), Easterly (2006), Alesina, Özler, Roubini, \& Swagel (1996), Rodríguez \& Rodrik (2000) y Barro \& Ursúa $(2008,2009)$.

El uso de datos de corte transversal de muestras grandes de países ha sido un enfoque determinante para identificar comportamiento o tendencias en todo el mundo, y América Latina no ha sido la excepción. La literatura empírica sobre crecimiento económico latinoamericano es abundante como lo demuestran Halperin (1961), Dagum (1964), Correa (1970), Mattos (1981), Tilak (1989), Cottani, Cavallo, \& Khan (1990), Gregorio (1992), Cardoso \& Fishlow (1992), Damil, Fanelli, Frenkel, Rozenwurcel, \& Wolfson (1993), Hutchinson \& Schumacher (1997), Cáceres \& Sandoval (1999), Chen (1999), Thornton (2001), Fernández-Arias \& Montiel (2001), Cunado, Gil-Alana, \& Gracia (2007), Muga \& Santamaría (2007), Blyde, Daude, \& Fernández-Arias (2010), Rhodes-Purdy (2017), Díaz \& Navarrete (2017). Para entender cuáles variables macroeconómicas contribuyeron al crecimiento económico latinoamericano, se adelantó una revisión de literatura a través de la cual se identificaron las siguientes: crecimiento del stock de capital, formación bruta de capital, crecimiento de la población, gasto de consumo final del gobierno general, exportaciones de bienes y servicios y nivel de inflación. Como consecuencia, el objetivo del artículo es determinar el impacto de las variables macroeconómicas identificadas en el crecimiento económico de América Latina durante el periodo de 1900 a 2000.

Este artículo se divide en tres secciones además de introducción y conclusión, la primera hace una exposición sobre los principales aportes empíricos a las teorías de crecimiento económico; la segunda detalla las fuentes de los datos y la metodología econométrica a realizar; en la última sección se muestran los resultados del trabajo empírico realizado con su respectivo análisis.

\section{REVISIÓN DE LITERATURA}

\section{Crecimiento general, convergencia absoluta y relativa}

La literatura empírica sobre crecimiento económico es abundante y puede tener variedad de acercamientos; sin embargo, su núcleo se encuentra en identificar la relación causal entre las variables elegidas y el impacto que tienen a lo largo del tiempo en una muestra representativa. Un trabajo importante es el de Robert Barro en 1991 que tiene como objetivo encontrar algunas regularidades empíricas sobre crecimiento, fertilidad e 
inversión para 98 países durante 1960-1985; sus principales resultados son: el crecimiento del PIB real per cápita está relacionado positivamente con los niveles iniciales de capital humano, y negativamente con el nivel inicial de PIB real per cápita; países con mayores niveles de capital humano exhiben menores tasas de fertilidad, y mayor proporción de inversión en capital físico con respecto al producto total; el crecimiento tiene una relación negativa con el gasto del gobierno general como proporción del producto; existe una relación despreciable entre crecimiento e inversión pública; por último, la estabilidad política de un país trae mayores niveles de crecimiento.

Por su lado, Cáceres \& Sandoval (1999) investigan la convergencia de 17 países latinoamericanos de 1950 a 1990; los autores muestran una convergencia en el ingreso per cápita después de 1979 pero con una gran inestabilidad en su tasa de crecimiento. Esta inestabilidad es explicada por la variabilidad en la acumulación de capital y del gasto público, como se mostrará más adelante. Cunado et al. (2007) tambien analizan el tema de la convergencia; su muestra se compone de once países emergentes de América Latina y Asia; su metodología se basa en un proceso de estimación paramétrica con el logaritmo del producto interno bruto real como variable endógena, así, concluyen diferentes de estados estacionario para cada economía.

A partir de estas investigaciones se puede concluir que el proceso de convergencia, en modelos de crecimiento exógeno, es resultado de dotaciones de tecnologías idénticas y exógenas para cada país. De esta forma, se asegura que la convergencia, ya sea absoluta o relativa, dependa de los rendimientos decrecientes a escala del capital y el radio de capital por trabajador; así, una acumulación de capital más rápida tendrá como consecuencia una tasa de crecimiento económico más alta.

Un último punto a resaltar sobre el crecimiento económico general son las trampas de pobreza o aceleraciones que sufren un grupo de países. En cuanto a trampas de pobreza, Easterly (2006) muestra que hay poca evidencia sobre países con tasa de crecimiento cero durante largos periodos de tiempo, y de igual forma, los despegues rápidos de crecimiento en los países pobres también son pocos comunes. Por su lado y a partir de una muestra de 110 países, Hausmann, Pritchett, \& Rodrik (2005) concluyen que las aceleraciones son comunes y no están acompañadas de cambios en las políticas económicas, condiciones institucionales o ambiente externo; simplemente son resultado del desenvolvimiento específico de una economía. En conclusión, no hay consenso sobre la capacidad que tiene un sistema para exhibir tasas de crecimiento fuera de su tendencia; sin embargo, el proceso de acumulación de capital y la formación bruta de capital se han logrado identificar como variables centrales en la explicación de crecimiento de las naciones.

\section{Factores y productividad}

Los factores de producción de capital y trabajo han sido el eje para la mayoría de trabajos empíricos sobre crecimiento económico; sin embargo, los análisis en este campo se han caracterizado por incluir variedad de aproximaciones intelectuales. Barro (1999) argumenta que las relaciones de las tasas de crecimiento de los factores, las participaciones de los factores, el cambio tecnológico (residuo econométrico), las políticas gubernamentales, las preferencias de los hogares, la disponibilidad de recursos naturales y los niveles iniciales de capital físico y humano son elementos clave para cualquier 
investigación. De igual forma, las crisis financieras y la política monetaria predominante también son determinantes a la hora de explicar el crecimiento en el producto de un país (Rivera \& Rivera, 2019).

Teniendo en cuenta lo anterior, Barro (2001) concluye que el crecimiento económico está positivamente relacionado con los niveles de educación primaria y secundaria de su población. Por su lado, Blyde et al. (2010), a partir de un panel de 76 países desarrollados en vía de desarrollo para el periodo 1960-2004, llegan a la conclusión que los colapsos del producto (caídas de la productividad total de los factores muy por debajo de su tendencia) son predominantes y contribuyen a una tasa de crecimiento más baja a largo plazo; además muestran que una disminución de la productividad lleva a una disminución del $12 \%$ en el producto interno bruto. Lo anterior confirma la preocupación por de las políticas enfocadas a mejorar el acervo de capital por trabajador y a mejorar su productividad.

En cuanto a la cantidad de trabajadores en una economía y el crecimiento poblacional se destaca como aporte principal artículo de Thornton (2001). El autor realiza un test de raíz unitaria y de causalidad, para examinar la relación de largo plazo entre la población y el PIB per cápita en siete países latinoamericanos, a lo largo de todo el siglo XX; de esta forma, Thornton concluye que no existe relación de largo entre ambas variables ya que el crecimiento poblacional no causa crecimiento en el ingreso por habitante. Este aporte es clave, pero no representa una conclusión general y se requieren de más estudios.

Por su lado, Hutchinson \& Schumacher (1997) se enfocan en la relación entre el gasto público y el crecimiento. Muestran que existe una relación positiva entre los gastos fiscales y el crecimiento económico en la forma de gasto en bienes públicos, de mérito y transferencias; estas pruebas apoyan la Ley de Wagner. En ella se postula que existe una correlación positiva entre el crecimiento económico per cápita y el gasto público. Hernández, (2005) muestra tres factores que justifican esta relación: 1) Por desplazamientos del sector privado ante un incremento en el gasto del estado 2) Por incrementos en los gastos de educación pública y políticas redistributivas del ingreso 3) Por posesión de monopolios económicos por parte del Estado.

En teoría, la mayor parte del gasto o inversión pública debe ser destinado a la provisión de bienes públicos como infraestructura ya que mejora la productividad de los factores productivos tal como lo demuestran Imran \& Niazi (2011), Gramlich (1994), Esfahani \& Ramírez (2003), Ramírez (2007) y Jaramillo, Meisel, \& Ramírez (2017).

\section{Económica política, instituciones y desigualdad}

El campo de la economía política y el impacto del arreglo institucional sobre el crecimiento económico ha tenido un desarrollo acelerado en los últimos 25 años; fruto de ello hay abundante literatura sobre el tema. El trabajo de Alesina et al. (1996) sirve como guía para familiarizarse con los principales aportes en el campo; los autores hacen una revisión de la literatura de la economía política del crecimiento económico destacando las dos vertientes de la nueva economía política y las teorías de crecimiento endógeno; de igual forma, los autores identifican en su análisis cuatro variables clave: crecimiento del producto interno bruto, acumulación de capital, inestabilidad política, libertad política, instituciones democráticas, y desigualdad en el ingreso. 
Similarmente, Alesina \& Perotti (1994) investigan la relación entre la inestabilidad política ${ }^{1}$ y el crecimiento del producto per cápita en un panel de 113 países para el periodo de 1950 a 1982; sus resultados muestran que la inestabilidad política reduce el crecimiento económico de manera significativa. Alesina \& Rodrik (1994) estudian la relación entre las relaciones políticas y el crecimiento económico a partir de un modelo de crecimiento endógeno, basado en el modelo de Romer de 1990, con la introducción de conflictos distributivos mediante diferentes proporciones de capital sobre trabajo. Los autores concluyen que la desigualdad en la propiedad de la tierra y en el ingreso están correlacionadas negativamente con el crecimiento económico.

El análisis de Barro (1996) muestra resultados contrarios a los anteriores. A partir de un panel de 100 países desde 1960 a 1990, el autor muestra que existe una relación negativa entre crecimiento económico y la democracia, una vez que se han controlado variables como mantenimiento del estado de derecho, los mercados libres, el pequeño consumo del gobierno y el nivel capital humano. Barro explica esta relación argumentando que existe una relación no lineal donde mayores niveles de democracia aumentan el rendimiento económico a bajos niveles de libertad política, pero frena el crecimiento una vez se han alcanzado niveles de libertad más altos. De forma similar, el mismo Barro (2000) encuentra una relación leve entre la desigualdad del ingreso y las tasas de crecimiento e inversión; más aún identifica una regularidad empírica donde una mayor desigualdad retardada el crecimiento económico en los países pobres, pero lo acelera en los ricos.

Específicamente para América Latina, Altman \& Castiglioni (2009) realizan un estudio de datos panel con 18 países para periodo 1972 a 2001 con el objetivo de examinar qué variables tienen impacto sobre el desarrollo humano; los autores concluyen que entre más democrática sea una sociedad, mejor será su rendimiento en términos de desarrollo humano. Esto confirma la urgencia que tienen los países de la región de solucionar las falencias en desigualdad de distribución del ingreso, pobreza, nutrición deficiente, mal acceso a la salud y cultura democrática. Por último, vale la pena destacar los trabajos de Panizza (2002) y Orozco \& Rivera (2018) que muestran la importancia de las instituciones en el desenvolvimiento económico y el trabajo de Rodrik \& Wacziarg (2005) sobre la importancia de la democracia.

\section{Comercio exterior}

La región latinoamericana ha orientado sus actividades comerciales hacia la especialización en actividades primarias-extractivas y de ensamble; fortaleciendo la actividad importadora. De esta forma, el sector exportador no ha podido constituirse como un generador de actividad productiva y se ha visto afectado por la volatilidad en la tasa de cambio y los cambios arbitrarios en políticas comerciales. Chen (1999), partir de una variación del modelo de crecimiento endógeno de Romer, concluye que las diferencias de crecimiento del último cuarto del siglo XX entre los países de Asia oriental con Latinoamérica se deben al grado de apertura al comercio exterior; es decir, más comercio atrae a más crecimiento. Por su lado, Cottani et al. (1990) analizan la relación entre la variabilidad del índice de la tasa real de cambio (ITRC) con el crecimiento del PIB real per cápita con el uso de un panel de datos de países menos desarrollados; los autores concluyen que existe una relación negativa y significativa entre la inestabilidad, la

\footnotetext{
${ }^{1}$ Esta variable se define como la probabilidad que tiene algún gobierno a colapsar.
} 
desalineación de la ITRC y los indicadores desempeño económico. Respecto a la tasa de cambio, Rodrik (2008) concluye que la devaluación, en países en vía de desarrollo estimula el crecimiento económico a través de los bienes no transables, especialmente la industria.

Específicamente para América Latina, Díaz \& Navarrete (2017) revisan la hipótesis de restricción externa de Thirwall y la condición Marshall-Lerner para Argentina, Brasil, Chile, Colombia y México durante el periodo de 1994 a 2015. Los autores llegan a los siguientes resultados: 1) La demanda del sector exportador y determina, en mayor medida, la trayectoria de crecimiento de una economía 2) Una apreciación en el tipo de cambio mejora la balanza comercial. En cuanto a la integración y posibilidad de formar un bloque comercial común, el economista argentino Camilo Dagum fue partidario y defensor de la integración comercial en América Latina, y en 1964 expone que la integración comercial es la única solución a los problemas en la propiedad de la tierra, dificultades en el proceso de industrialización y necesidad de divisas para reservas internacionales. A igual que Dagum, Rivera-Batiz \& Romer (1991) resaltan los efectos positivos de la integración económica de una región en la forma de flujo de ideas y tecnología, infraestructura común y políticas comerciales y tributarias comunes. Los dos resultados anteriores apoyan el estudio empírico realizado por Naveed \& Shabbir, (2006) donde muestran que la apertura está positivamente relacionada con el crecimiento del PIB per cápita.

Un punto de vista opuesto al anterior es el de Fernández-Arias \& Montiel (2001), los autores argumentan que el crecimiento económico de América Latina no ha sido más alto después de las reformas estructurales de los años noventa; esto se debe a un ambiente internacional no propicio y a deficiencias institucionales y productivas internas.

\section{METODOLOGÍA Y DATOS}

Los países de la muestra son Argentina, Bolivia, Brasil, Chile, Colombia, Costa Rica, Cuba, República Dominicana, Ecuador, El Salvador, Guatemala, Haití, Honduras, México, Nicaragua, Panamá, Paraguay, Perú, Uruguay, Venezuela; el periodo de estudio es 1900 2000. Los datos a utilizar son recopilados en los proyectos: 1) Maddison Project Database (MPD) que abarca el periodo de 1900-2000 y su documentación se encuentra en Bolt, Inklaar, Jong, \& Zanden (2018) 2) Penn World Table 9.0 (PWT) abarca el periodo de 19502000 y su documentación se encuentra en Feenstra, Inklaar, \& Timmer (2015). Ambas bases de datos son construidas por el Centro de Crecimiento y Desarrollo de Groningen ${ }^{2}$ (GGDC por sus siglas en inglés) fundado en el Departamento de Economía de la Universidad de Groningen en 1992.

Teniendo en cuenta lo anterior, las variables elegidas son:

1. PIB real per cápita: expresado en dólares estadounidenses de 2011.

2. PIB real per cápita (TC): esta variable tiene como objetivo comparar tasas de crecimiento relativas entre países durante un periodo largo de tiempo. Es una medida del PIB real per cápita usando tasas de crecimiento de cuentas nacionales y está expresada en dólares estadounidenses de 2011 (Bolt et al., 2018).

${ }^{2}$ La página de este centro de estudios es https:/www.rug.nl/ggdc/. En este enlace se pueden encontrar todas las bases de datos disponible con su respectiva documentación. 
3. Formación bruta de capital: consiste en desembolsos por adiciones a los activos fijos de la economía más los cambios netos en el nivel de inventarios (Feenstra et al., 2015). Lorente (2018) argumenta la formación bruta de capital se puede adoptar como la inversión bruta del período ya que representa el capital contable de las empresas. En base a esto, se usa la formación de capital como equivalente a la inversión bruta.

4. Stock de capital: son los bienes durables usados en la producción como maquinaria, equipos e infraestructura; está expresada en dólares estadounidenses de 2011 (Feenstra et al., 2015).

5. Gasto de consumo final del gobierno general: incluye todos los gastos corrientes del gobierno para compra de bienes y servicios (Feenstra et al., 2015).

6. Exportaciones de bienes y servicios: representan el valor de todos los bienes y otros servicios de mercado prestados al resto del mundo.

Con un simple cálculo se puede obtener las tasas de crecimiento de las respectivas variables:

$$
\text { [1] Tasa de crecimiento de Variable } \left.\text { Variable }_{t}-\text { variable }_{(t-1)}\right) * 100
$$

Gracias a la ecuación [1] se obtiene la tasa de crecimiento del PIB real per cápita a partir de la serie PIB real per cápita (TC) y la tasa de crecimiento del Stock de capital y de la población a partir de los datos originales.

La última variable elegida es la variación en el nivel de precios; los datos sobre la inflación son obtenidos gracias al trabajo de Carmen Reinhart ${ }^{3}$ y Kenneth Rogoff. La serie de tiempo es obtenida específicamente de Reinhart \& Rogoff (2011) y abarca el periodo de 1900-2000 con algunas excepciones.

La metodología utilizada es llevar a cabo regresiones lineales simples de mínimos cuadrados ordinarios (MCO) combinados sin punto de corte. De esta forma la variable endógena es la tasa de crecimiento anual porcentual del PIB real per cápita (gY_cápita) y las variables exógenas son crecimiento del stock de capital (gK), formación bruta de capital (FBK), crecimiento poblacional (gPob), gasto de consumo final del gobierno general $(G)$, exportaciones de bienes y servicios (E) e inflación $(\pi)$. Debido a la cantidad de variables se realizan cinco regresiones para entender cuál es la relación entre la variable endógena y la variable exógena bajo distintos escenarios. Cabe señalar que el objetivo de este documento no es realizar un trabajo econométrico exhaustivo y sofisticado, sino entender de manera exitosa la relación entre variables. Así pues, los modelos a obtener son:

$$
\begin{aligned}
& g Y_{-} \text {ápita }_{i t}=g K_{i t}+g P o b_{i t}+\pi_{i t}+U_{i t} \\
& g Y_{-} \text {ápita }_{i t}=g K_{i t}+F B K_{i t}+\pi_{i t}+U_{i t} \\
& g Y_{-} \text {ápita }_{i t}=F B K_{i t}+G_{i t}+E_{i t}+U_{i t} \\
& g Y_{-} \text {ápita }_{i t}=G_{i t}+E_{i t}+g P o b_{i t}+U_{i t}
\end{aligned}
$$$$
\text { [1] }
$$

\footnotetext{
${ }^{3}$ En este enlace se puede encontrar todas las bases de datos realizadas por los autores http://www.reinhartandrogoff.com/
} 


$$
g Y_{-} \text {cápita }_{i t}=g K_{i t}+F B K_{i t}+G_{i t}+E_{i t}+g P o b_{i t}+\pi_{i t}+U_{i t}
$$

Donde $i$ representa al país, $t$ representa el tiempo $(t=1900, \ldots, 2000)$ y $U_{i t}$ los errores del modelo.

\section{RESULTADOS}

El ingreso por habitante en la región pasó de 1365 dólares en el año 1900 a 8728 dólares en el 2000 con una tasa de crecimiento promedio de 1,83\% anual y desviación estándar de 2,7115\%, específicamente en la segunda mitad del siglo se observó una tasa de crecimiento de 1,96\% a diferencia del crecimiento mundial que fue de 2,33\%. En los países de la muestra se identificaron 1775 observaciones con crecimiento per cápita negativo de un total de 2020. Lo anterior demuestra que en el $87,87 \%$ de los años analizados, los países de la muestra tuvieron tasa de crecimiento negativas; sin embargo, los datos del ingreso por habitante muestran que el ingreso latinoamericano paso a ser 6,40 veces a final. Teniendo en cuenta lo anterior es válido preguntar ¿Cómo logro Latinoamérica aumentar su ingreso teniendo tantos años con tasas de crecimiento negativas?

La respuesta de la pregunta anterior se encuentra en la variación que mostró el proceso de crecimiento latinoamericano; la baja desviación estándar mostrada en el párrafo anterior es resultado de promediar las desviaciones estándar de cada país; sin embargo, si se subdivide aún más la muestra se puede observar una situación totalmente diferente. Argentina tuvo una tasa de crecimiento del ingreso per cápita negativa de 8,012\% en 1959 y al año siguiente creció positivamente a 6,06\%; Uruguay pasó de $-2,56 \%$ a 9,20\% en 1953 y Ecuador de 10,628\% a -1,03\% en 1949. Sucesos como los anteriores fueron comunes en el siglo XX; se puede concluir, entonces, que los años de crecimiento positivo lograron absorber los choques provocados por los años de crecimiento negativo. De igual forma el aumento en la productividad por trabajador debió aumentar a lo largo del siglo por tres razones principales: 1) El stock de capital de los países de la región aumento de forma sostenida durante todo el periodo de estudio 2) Las variaciones de la tasa de crecimiento positivo fueron demasiado altas debido a la introducción de nuevas tecnologías o procesos productivos 3) El ingreso real per cápita aumento a pesar de exhibir tasas de crecimiento poblacional elevadas.

Ahora bien, en la tabla 1 se encuentran los resultados obtenidos, específicamente los coeficientes de regresión con su respectiva significancia, coeficiente de determinación $\left(R^{2}\right)$ y coeficiente de determinación ajustado. Es importante señalar que el siguiente análisis se hace solo en base a las variables que resultaron significativas al 5\% y $10 \%$ para garantizar la validez de las conclusiones.

Crecimiento del Stock de capital: En promedio, por cada 1\% que aumentó el crecimiento del stock de capital, se estima que el crecimiento del producto interno bruto per cápita aumentó $0,5489 \%$. El crecimiento promedio del stock de capital en los países de la muestra fue de 4,083\% con una desviación estándar de 2,247\% de 1950 a 1975; y un crecimiento de 4,18\% con una desviación de 2,328\% de 1975 a 2000. El límite superior de las desviaciones estándar muestra que la región mantuvo un crecimiento del stock del capital estable y acorde con su nivel de desarrollo. 
Tabla 1: Resultados de modelos econométricos

\begin{tabular}{|c|c|c|c|c|c|}
\hline \multicolumn{6}{|c|}{ Estimaciones de MCO de efectos sobre el crecimiento económico por habitante } \\
\hline \multicolumn{6}{|c|}{ Variable Dependiente: Crecimiento del producto interno bruto per cápita (\%) 1900-2000 } \\
\hline & (1) & $(2)$ & (3) & (4) & $(5)$ \\
\hline Crecimiento del Stock de capital & $0,5547^{\star}$ & $0,5394^{\star}$ & & & $0,5527^{*}$ \\
\hline Formación bruta de capital & & $-0,0238^{\star *}$ & $0,1106^{*}$ & & 0,0140 \\
\hline $\begin{array}{l}\text { Gasto de consumo final del } \\
\text { gobierno general }\end{array}$ & & & $-0,0479 *$ & 0,0097 & $-0,0431^{\star}$ \\
\hline Exportaciones de bienes y servicios & & & 0,0164 & $0,0307^{*}$ & 0,0161 \\
\hline Crecimiento poblacional & $-0,2342^{*}$ & & & $0,3269 *$ & $-0,1750$ \\
\hline Inflación & $-0,0007^{*}$ & $-0,0007^{\star}$ & & & $-0,0006^{*}$ \\
\hline$R^{2}$ & $27,93 \%$ & $27,49 \%$ & $15,56 \%$ & $10,41 \%$ & $28,44 \%$ \\
\hline$R^{2}$ ajustado & $27,69 \%$ & $27,24 \%$ & $15,3 \%$ & $10,13 \%$ & $27,96 \%$ \\
\hline
\end{tabular}

Específicamente, Brasil, Chile, Colombia, Costa Rica, México, Panamá, República Dominicana y Venezuela lograron mantener un crecimiento en esta variable superior al $4 \%$ durante toda la segunda mitad del siglo XX; lo que significa que, en promedio, cada uno de estos países logro duplicar su acervo de capital cada 25 años. Los resultados anteriores confirman la importancia que tiene el proceso de acumulación de capital en el crecimiento económico de los países; y pone de manifiesto la necesidad de implementar políticas o regulaciones encaminadas a facilitar el proceso de acumulación. Lo anterior no quiere decir, de ninguna forma, que el gobierno deba ser el encargado de llevar a cabo este proceso; simplemente debe facilitar las condiciones para que los demás agentes de la sociedad puedan llevarlo a cabo exitosamente.

Formación bruta de capital: esta variable tuvo un efecto positivo promedio de 0,0434\% en el crecimiento del ingreso por habitante. Durante la segunda mitad del siglo XX, la participación promedio de la inversión bruta en el producto interno bruto latinoamericano de 17,88\% con una desviación estándar de 3,852\%. Los países de Colombia, Ecuador, México, Panamá, República Dominicana y Venezuela tuvieron tasas superiores al $20 \%$ durante los años con disponibilidad de datos. Al realizar un análisis de heterogeneidad de las desviaciones de cada país, se encuentra que este rubro fue relativamente variable; destacando a Panamá con 5,75\%, Republica Dominicana con $5,835 \%$ y Venezuela con 9,88\%. La variación de la inversión bruta del periodo se puede explicar por las elecciones que los individuos deben realizar entre consumir e invertir; una economía, con recursos domésticos, no se puede volcar solamente a invertir ya que el consumo se contraería fuertemente y no habría recursos para invertir en periodos posteriores. Más aún, las inversiones en maquinarias e infraestructura no son tangibles inmediatamente, y requieren de tiempo para ser puestas en marcha; lo anterior puede limitar aún más el impacto en el corto plazo que tienen la formación bruta de capital en el crecimiento. A la luz del análisis anterior; los países latinoamericanos deben lograr aprovechar el ahorro del resto de mundo para aumentar sus inversiones y así lograr un nivel de crecimiento más acelerado.

Gasto de consumo final del gobierno general: en promedio, por cada 1\% que aumente esta variable, se estima que el crecimiento del PIB disminuye 0,0455\%. La participación 
promedio del gasto de consumo final del gobierno general fue de $14,565 \%$ con una desviación estándar de 5,60\%. Específicamente, Bolivia, Chile, Costa Rica, Ecuador, El Salvador, Panamá, Perú, Uruguay y Venezuela mantuvieron una participación del gasto púbico dentro del producto superior al 15\% durante toda la segunda mitad del siglo XX. La naturaleza política e ideológica hace que el gasto público no sea capaz de proporcionarle a una economía crecimiento constante; más aún, una política fiscal errónea puede llevar un país a entrar en un default, una espiral inflacionaria o contraer de tal manera las libertades individuales que el desarrollo económico sea imposible. A partir de los resultados y de la revisión de literatura, se puede concluir que el gasto público es una herramienta para reducir las fricciones sociales generadas por la desigualdad en el ingreso y en el acceso a la tierra; y no una herramienta cuyo objetivo principal sea impulsar la demanda agregada. En ese orden de ideas, el gasto público debe especializarse en la provisión de bienes públicos puros que no atenten contra el equilibrio macroeconómico del país.

Exportaciones de bienes y servicios: en promedio, por cada 1\% que aumenten los bienes y servicios exportados, se estima que existe un efecto positivo en el crecimiento de 0,0307\%. La participación promedio de las exportaciones fue de 15,232\% con una desviación estándar de 4,4255\%. Los países de Bolivia, Chile, Costa Rica, Ecuador, El Salvador, Guatemala, Haití, Honduras, Panamá, Paraguay, Republica Dominicana y Venezuela lograron mantener esta variable por encima del 12\% durante 1950-2000. Por otro lado, Bolivia, El Salvador, Republica Dominicana y Venezuela tuvieron importaciones superiores al $20 \%$ del producto para el mismo periodo de tiempo. Los resultados anteriores confirman las hipótesis presentadas en la sección de revisión de literatura que exponían que el comercio exterior tiene un impacto positivo sobre el crecimiento. Sin embargo, el resultado anterior se debe complementar con estudios sectoriales sobre el impacto que tiene la apertura económica sobre empresas nacionales específicas y no solo en el agregado. En conclusión, el comercio exterior beneficia, innegablemente, a todos los agentes que participan en él, pero se deben hacer análisis complejos, más allá de elasticidades, a la hora de decidir el camino de las políticas públicas relacionadas.

Crecimiento poblacional: en promedio, por cada $1 \%$ que aumente estas variables, se estima que el crecimiento del PIB per cápita aumento 0,04635\%. El crecimiento poblacional promedio de los países de la muestra fue de 1,88\% con una desviación estándar de 0,89\% para 1900 a 1950. Mientras que para la segunda mitad del siglo se tuvo un crecimiento de 2,389\% con una desviación estándar de 0,844\%; a diferencia del crecimiento de 2,58\% con desviación de 5,49\% que experimento el mundo para el mismo periodo. En cifras brutas, la región pasó de tener 62662000 habitantes en 1900 a 516150000 en el año 2000; la población paso a ser 8,2 veces mayor y aun así logro materializar aumentos en su nivel de vida tal como se vio al inicio de esta sección. Para este caso, los resultados de los modelos realizados no son claros ya que en el modelo 1 el coeficiente resultante fue $-0,2342$ y en el modelo 4 la beta resultante fue 0,3269; lo anterior confirma la relación confusa que existe entre crecimiento económico y crecimiento poblacional; se deben realizar más investigaciones para llegar a un acuerdo común sobre esta relación.

Inflación: se estima que, en promedio, un aumento de un punto porcentual en la tasa inflación tiene un efecto negativo sobre la variable dependiente igual a 0,0006\%. En los 
países de la muestra se registraron 191 episodios de deflación anual; específicamente Colombia registro los perores episodios de deflación con -29,73\% en 1921, -29.411\% en 1902 y -23,82\% en 1931, Venezuela también experimentó una tasa de inflación negativa superior al 20\% con 25,21\% en 1921. Se observaron 1209 episodios de inflación positiva, sin embargo, se hace necesario clasificar los datos aún mejor. Así, se registraron: 22 episodios en los cuales la tasa de inflación anual fue superior al 500\%; 46 casos con inflación mayor a 100\% y menor o igual al 500\%; 68 casos con variación en el nivel de precio entre 50\% y 100\%; 519 episodios con inflación mayor a 10\% y menor a 50\%; y finalmente, 584 episodios con tasa de inflación positiva menor a 10\%.

Los países que tuvieron inflaciones promedio decenales menores al promedio muestral fueron Colombia, Costa Rica, Ecuador, El Salvador, Guatemala, Honduras, México, Panamá, República Dominicana Venezuela. En el lado opuesto del espectro se encuentran Argentina y Brasil con inflación crónica. En conclusión, los países del primer grupo han tenido una política monetaria exitosa mientras que el manejo que le han dado los segundos ha sido deficiente; la importancia para el crecimiento de mantener una inflación baja y estable radica en que facilita la asignación eficiente de los recursos escasos, disminuye la incertidumbre, no interfiere en los incentivos para llevar a cabo el proceso de acumulación de capital y protege los contratos financieros entre individuos.

\section{CONCLUSIONES}

El presente artículo logró determinar el impacto de las variables macroeconómicas identificadas en el crecimiento económico de América Latina durante el siglo XX. Además, gracias a la revisión de literatura realizado se logró establecer la explicación causal que juega cada una de estas variables el crecimiento económico bajo la visión de crecimiento por acumulación de factores, por innovación e hipótesis institucionales.

En primer lugar, el crecimiento del stock de capital y la formación bruta de capital son variables centrales para explicar el proceso de acumulación de capital y el aumento de capacidad productiva en cualquier economía. Segundo, el gasto del gobierno general como proporción del PIB mostró una relación negativa con el crecimiento del ingreso real per cápita; sin embargo, el gasto público es clave pasa solucionar conflictos distributivos y sociales. Por lo anterior, el gasto debe ser cada vez más técnico y científico dejando de lado los gastos ideológicos y políticos.

En tercer lugar, el comercio exterior es una herramienta clave para que cualquier país logre beneficiarse de las ventajas comparativas, financiamiento exterior, flujo de ideas y de bienes del resto del mundo. Cuarto, el crecimiento poblacional continúa siendo una variable que no muestra una relación clara con el crecimiento económico y se requieren de más estudios para la región; a pesar de lo anterior, la conclusión de este artículo es que existe una relación positiva y significativa entre crecimiento económico y crecimiento poblacional. Por último, la sostenibilidad inflacionaria es la única capaz de proveer un entorno con mínima incertidumbre para el desenvolvimiento económico de los individuos. 


\section{REFERENCIAS}

Acemoglu, D., \&amp; Robinson, J. (2010). The Role of Institutions in Growth and Development. Review of Economics and Institutions, 1(2).

Acemoglu, D., Johnson, S., \&amp; Robinson, J. (2001). The colonial origins of comparative development: an empirical investigation. The American Economic Review, 97(5), 1369-1401.

Aghion, P., Howitt, P. (1992). A Model of Growth through Creative Destruction. Econometrica, 60(2), 323-351.

Alesina, A., Perotti, R. (1994). The Political Economy of Growth: A Critical Survey of the Recent Literature. The World Bank Economic Review, 8(3), 351-371.

Alesina, A., Rodrik, D. (1994). Distributive Politics and Economic Growth. The Quarterly Journal of Economics, 109(2), 465-490.

Alesina, A., Özler, S., Roubini, N., Swagel, P. (1996). Political Instability and Economic Growth. Journal of Economic Growth, 1(2), 189-211.

Altman, D., Castiglioni, R. (2009). Democratic Quality and Human Development in Latin America: 1972-2001. Revue canadienne de science politique, 42(2), 297-319.

Barro, R. (1991). Economic Growth in a Cross Section of Countries. The Quarterly Journal of Economics, 106(2), 407- 443.

Barro, R. (1996). Democracy and Growth. Journal of Economic Growth, 11, 1-27.

Barro, R. (1999). Notes on Growth Accounting. Journal of Economic Growth, 4(2), 119-137.

Barro, R. (2000). Inequality and Growth in a Panel of Countries. Journal of Economic Growth, 5(1), 5 32.

Barro, R. (2001). Human Capital and Growth. The American Economic Review, 91(2), 12-17.

Barro, R., Ursúa, J. (2008). Rare Macroeconomic Crises since 1870. Brookings Papers on Economic Activity, 2008, 255-335

Barro, R., Ursúa, J. F. (2009). Stock-market crashes and depressions. NBER Working Paper No. 14760.

Blyde, J. S., Daude, C., Fernández-Arias, E. (2010). Output collapses and productivity destruction. Review of World Economics, 146(2), 359-387.

Bolt, J., Inklaar, R., Jong, H. d., Zanden, J. L. (2018). Rebasing 'Maddison': new income comparisons and the shape of long-run economic development. Maddison Project Working Paper 10. Obtenido de www.ggdc.net/maddison

Cáceres, L. R., Sandoval, Ó. N. (1999). Crecimiento económico y divergencia en la América Latina. El Trimestre Económico, 66(4), 781-796.

Cardoso, E., \&amp; Fishlow, A. (1992). Latin American Economic Development: 1950-1980. Journal of Latin American Studies, 24, 197-218.

Cass, D. (1965). Optimum Growth in an Aggregative Model of Capital Accumulation. Review of Economic Studies, 32, 233-240.

Chen, B.L. (1999). Trade Openness and Economic Growth: Evidence in East Asia and Latin America. Journal of Economic Integration, 14(2), 265-295.

Correa, H. (1970). Sources of Economic Growth in Latin America. Southern Economic Journal, 37(1), 17-31.

Cottani, J. A., Cavallo, D. F., Khan, M. S. (1990). Real Exchange Rate Behavior and Economic Performance in LDCs. Economic Development and Cultural Change, 39(1), 61-76.

Cunado, J., Gil-Alana, L., Gracia, F. P. (2007). Real convergence in some emerging countries: a fractionally integrated approach. Recherches Économiques de Louvain, 73(3), 293-310.

Currie, L., Sandilands, R. (1997). "Implications of an endogenous theory of growth in Allyn Young's macroeconomic concept of increasing returns. History of Political Economy, 29(3), 413-443.

Dagum, C. (1964). La Integración y el Crecimiento Económico en América Latina. Desarrollo Económico, 4(15), 237-262. 
Damil, M., Fanelli, J. M., Frenkel, R., Rozenwurcel, G., Wolfson, L. (1993). Crecimiento económico en América Latina: Experiencia reciente y perspectivas. Desarrollo Económico, 33(130), 237-26.

Díaz, H. O., Navarrete, R. A. (2017). Crecimiento, competitividad y restricción externa en América Latina. Investigación Económica, 76(300), 53-80.

Easterly, W. (2006). Reliving the 1950s: The Big Push, Poverty Traps, and Takeoffs in Economic Development. Journal of Economic Growth, 11(4), 289-318.

Engerman, S., Sokoloff, K. (2002). Factor endowments, inequality, and paths of development among. Working paper 9259, NBER.

Esfahani, H. S., Ramírez, M. T. (2003). Institutions, infrastructure, and economic growth. Journal of Development Economics, 70(2), 443- 477.

Feenstra, R., Inklaar, R., Timmer, M. (2015). The Next Generation of the Penn World Table. American Economic Review, 105(10), 3150-3182. Retrieved from www.ggdc.net/pwt

Fernández-Arias, E., Montiel, P. (2001). Reform and Growth in Latin America: All Pain, No Gain? IMF Staff Papers, 3, 522-546.

Gramlich, E. (1994). Infrastructure Investment: A Review Essay. Journal of Economic Literature, 32(3), 1176-1196.

Gregorio, J. d. (1992). El crecimiento económico en la América Latina. El Trimestre Económico, 59, 75-107.

Halperin, M. (1961). Growth and Crisis in the Latin American Economy. Science Society, 25(3), 195228.

Hausmann, R., Pritchett, L., Rodrik, D. (2005). Growth Accelerations. Journal of Economic Growth, 10(4), 303-329.

Hernández, I. (2005). Teoría política y fiscal. Bogotá: Universidad Externado de Colombia.

Hutchinson, G. A., Schumacher, L. (1997). Fiscal expenditure policy and economic growth: Evidence from Latin America and the Caribbean. Social and Economic Studies, 46(4), 1-16.

Imran, M., Niazi, J. (2011). Infrastructure and Growth. The Pakistan Development Review, 50(4), 355 364.

Jaramillo, J., Meisel, A., Ramírez, M. T. (2017). More than One Hundred Years of Improvements in Living Standards: the Case of Colombia. Borradores de Economia 1027, Banco de la Republica de Colombia.

Koopmans, T. C. (1965). On the Concept of Optimal Economic Growth. Chicago, Rand McNally.

Lorente, L. (2018). Dinámica del crecimiento económico. Bogotá: Universidad Nacional de Colombia.

Mankiw, N. G., Phelps, E. S., Romer, P. (1995). The Growth of Nations. Brookings Papers on Economic Activity, (1), 275-326.

Mattos, C. A. (1981). Crecimiento y concentración espacial en la América Latina: algunas consecuencias. El Trimestre Económico, 48(2), 341-362.

Muga, L., Santamaría, R. (2007). The Momentum Effect in Latin American Emerging Markets. Emerging Markets Finance \& Trade, 43(4), 24-45.

Naveed, A., Shabbir, G. (2006). Trade openness, FDI and economic growth: A Panel Study. Pakistan Economic and Social Review, 44(1), 137-154.

Nunn, N. (2009). The Importance of History for Economic Development. Annual Review of Economics, 1, 65-92.

Orozco, C., Rivera, N. (2018). Instituciones como factor decisivo para el crecimiento económico. Econografos - Escuela de Economía (120).

Panizza, U. (2002). Income Inequality and Economic Growth: Evidence from American Data. Journal of Economic Growth, 7(2), 25-41.

Ramírez, M. T. (2007). Efectos de Eslabonamiento de la Infraestructura de Transporte sobre la Economía Colombiana: 1900-1950. In J. Robinson, M. Urrutia, Economía colombiana del siglo XX: Un análisis cuantitativo (pp. 383-457). Fondo de Cultura Económica. 
Reinhart, C., Rogoff, K. (2009). The Aftermath of Financial Crises. The American Economic Review, 99(2), 466-472.

Reinhart, C., Rogoff, K. (2009). This time is different: eight centuries of financial folly. Princeton University Press.

Reinhart, C., Rogoff, K. (2011). From Financial Crash to Debt Crisis. American Economic Review, 101(5), 1676-1706.

Rhodes-Purdy, M. (2017). Beyond the Balance Sheet: Performance, Participation, and Regime Support in Latin America. Comparative Politics, 49(2), 252-272.

Rivera Lozano, M., Rivera Garzón, N. (2019). Crisis financieras: esta vez no es distinto. Revista Finanzas y Política Económica, 11(1).

Rivera-Batiz, L. A., Romer, P. (1991). Economic Integration and Endogenous Growth. The Quarterly Journal of Economics, 106(2), 531-555.

Rodríguez, F., Rodrik, D. (2000). Trade Policy and Economic Growth: A Skeptic's Guide to the CrossNational Evidence. NBER Macroeconomics Annual, 15(2000), 261-325.

Rodrik, D. (2008). The Real Exchange Rate and Economic Growth. Brookings Papers on Economic Activity, 365-412.

Rodrik, D., Wacziarg, R. (2005). Do Democratic Transitions Produce Bad Economic Outcomes? The American Economic Review, 95(2), 50-55.

Romer, P. (1990). Endogenous technological change. Journal of Political Economy, 98(5), 71-102.

Schumpeter, J. (1911). The theory of economic development. Cambridge: Harvard University Press.

Slemrod, J., Gale, W., Easterly, W. (1995). What Do Cross-Country Studies Teach about Government Involvement, Prosperity, and Economic Growth? Brookings Papers on Economic Activity, (2), 373431.

Solow, R. (1956). A contribution to the theory of economic growth. Quarterly Journal of Economics, 70, 65-94.

Thornton, J. (2001). Population Growth and Economic Growth: Long-Run Evidence from Latin America. Southern Economic Journal, 68(2), 464-468.

Tilak, J. (1989). The Recession and Public Investment in Education in Latin America. Journal of Interamerican Studies and World Affairs, 37(2), 125-146.

Wolf, H. (1994). Growth Convergence Reconsidered. Weltwirtschaftliches Archiv, 130(4), 747-759.

Young, A. (1928). Increasing Returns and Economic Progress. The Economic Journal, 38(152), 527542. 